International Journal of Mechanical Engineering and Technology (IJMET)

Volume 12, Issue 4, April 2021, pp. 25-35. Article ID: IJMET_12_04_002

Available online at https://iaeme.com/Home/issue/IJMET?Volume $=12 \&$ Issue $=4$

ISSN Print: 0976-6340 and ISSN Online: 0976-6359

DOI: https://doi.org/10.34218/IJMET.12.4.2021.002

(C) IAEME Publication

Scopus Indexed

\title{
ANONYMIZATION OF SENSITIVE DATA IN UNSTRUCTURED DOCUMENTS USING NLP
}

\author{
Anushree Raj \\ Assistant Professor, Computer Science and Application, Reva University, \\ Bangalore, India \\ Rio D'Souza \\ Professor, Computer Science and Engineering, St Joseph Engineering College, \\ Mangalore, India
}

\begin{abstract}
Lot of researchers have worked for the progress of anonymization of structured data through spread-sheets and database tools. Masking of sensitive information in structured data and data anonymization is possible through algorithms or techniques. But anonymizing unstructured data is a real challenge since data currently exists in different form.

The study which ensures to cope with the interactions between human language and computers is called NLP. Natural Language Processing is the sub-field of AI which focuses on enabling computers to understand and process human languages. Further, we provide the deeper insight on how NLP works and show how a system can understand unstructured text, extract sensitive data and perform anonymization. The proposed anonymization procedure provides a system to apply text anonymization on unstructured original medical-records of an individual and release the anonymized document to help researchers for further study or investigation by preserving the privacy of the concerned individual.
\end{abstract}

Keywords: CRF, NE, Natural language Processing, unstructured data, anonymization.

Cite this Article: Anushree Raj and Rio D'Souza, Anonymization of Sensitive Data in Unstructured Documents Using NLP, International Journal of Mechanical Engineering and Technology (IJMET), 12(4), 2021, pp.25-35.

https://iaeme.com/Home/issue/IJMET?Volume=12\&Issue $=4$

\section{INTRODUCTION}

Artificial Intelligence is the most advancing field which has made machines to imitate the performance and capabilities of the human mind. It is been a long time where researchers are investing their plenty of work in integrating language into the field of artificial intelligence in the form of Natural Language Processing [1]. 
Through natural language processing, computers learn to perfectly deal with and relate overall linguistic sense to text excerpts like phrases or sentences [2]. The challenge involved is to summarize lengthy texts or target and extract specific keywords and information within a bulky content of text which requires a deep study of linguistic syntax at the current situation. The data available could be some private information which comprise of some sensitive information of an individual.

The best solution in this situation is to perform an anonymization process to mask the identities of actual entities exposed in any way by the text document. Sharing of textual data is very useful in proving necessary information and accurate knowledge for the researchers to get more insights from any textual document [3]. But the threat is only about sharing the sensitive information of an individual and violating the privacy preserving measures [4]. I we consider the medical records, which provides the information concerning the analysis and treatment of earlier patients which could be used as an reference to enlighten existing procedures and provide additional knowledge for further study or treatments. But these documents usually refer to original information of the real patients and hence must be anonymised prior to it is shared [5]. Accordingly, the data which is extensively useful for research purposes may be suspended to guard its authors sensitive information against adverse authorized and privacy preserving cause. The best possible alternative solution is to anonymize the sensitive information in the document using the technology of Natural Language Processing effectively.

Sharing information of any individual with third parties is a matter of privacy concern. In the U.S., the Health Insurance Portability and Accountability Act (HIPAA) [6] states that clinical data cannot be published unless it is de-identified. HIPAA specifies a list of seventeen categories of identifiers that should be removed prior to exposing a document to the public. Also, the General Data Protection Regulation (GDPR) [7] proposes a unified law of privacy protection on the processing of personal data and on the free circulation of such data, and it advises to take serious measures to preserve the privacy of personal data in order to avoid privacy breaches.

In our proposed work, we demonstrate an anonymization system based on a Named Entity Recognition (NER) tool for unstructured text documents. We assess the outcomes of few existing anonymization methods over text documents. This paper is divided into seven sections as follows: Section II gives the description of textual anonymzation problem, Section III gives an overview of the related work in the area of text anonymization. Section IV demonstrates the proposed anonymization system, describing the steps involved. Section V illustrates the results and evaluation. Finally, Section VI concludes the paper and lists some future work.

\section{TEXTUAL ANONYMIZATION PROBLEM}

\subsection{Anonymization}

The task is to extract the quasi-identifier and/or sensitive attributes from unstructured textual data. We focus to identify attributes such as patient name, location, birth date, etc. For the sake of concreteness, here we would focus on medical diagnosis reports. The key factor of text anonymization systems is to mask the sensitive data in medical records [8]. Anonymization of medical records is essential for publishing and using experimental data for research purposes without the risk of illegal access to the patients' identification [9]. Most of these anonymization systems have been applied to texts written in English. A standard anonymization system is generally composed of four modules: i) performs text normalization and feature extraction; ii) Named Entity (NE) classification; iii) distribution of entities into their respective class of NE; and iv) choose an anonymization method over the NEs and replaces the occurrences of these entities in the text. 


\subsection{Named-Entity Recognition}

Named Entity Recognition is a form of text mining that sieves through the unstructured text data and traces the noun phrases which are so called the named entities [10]. The named entities can then be structured under predefined class, such as "person," "organization," "disease," "date," or "number". NER is constructive in the anonymization of unstructured data which represents the free text documents. It can also identify all those expressions that may be used to re-identify an individual and those expressions that enclose sensitive information [11]. Once these terms have been located, anonymization methods like generalization, suppression, tagging etc. can be used to control the risk of re-identification of an individual sensitive data.

\subsection{Conditional Random Fields}

In natural language processing, the NER tasks can be solved by either of the two modes: hidden Markov models (HMMs) [12, 13], and conditional random fields CRFs [14, 15, 16]. CRFs are extensively used and functions in NER, and generally gives the best results in many fields, so in our proposed work we design our model using CRFs. CRFs [17] are provisionally trained undirected graph models often useful in pattern recognition.

\section{RELATED WORK}

The main use of sensitive data anonymization systems for unstructured data is to de-identify these unstructured records which could contain the sensitive and private information of an individual which could be made available to the third party on anonymizing this information. Anonymization of any personal records is critical to publish and use this data for research purposes without the risk of unauthorized access to the persons' identification.

Several techniques to anonymize unstructured textual data can be classified into either of these two categories: dictionary based techniques or machine learning techniques

\subsection{Replacing Personally-Identifying Information in Medical Records, the Scrub System}

Latanya Sweeney: Scrub [18] defines a new approach to locating and replacing personallyidentifying information in medical records that extends beyond straight search and-replace procedures, and we provide techniques for minimizing risk to patient confidentiality. Scrub uses detection algorithms that employ templates and specialized knowledge of what constitutes a name, address, phone number and so forth.

Once personally identifying information is detected, it must be replaced with some pseudovalue. There are several strategies for accomplishing this feat. Associated with each detection algorithm in Scrub is a replacement algorithm that is responsible for producing the replacement text. In general, the format of the replacement text matches the template that was recognized. If the detected entity was a date, for example, the replacement date may involve lumping days to the first of the nearest month or some other grouping. On the other hand if the detected entity was a first name, the typical strategy is to perform a hash-table lookup using the original name as the key. The result of the look-up is the replacement text. This provides consistent replacements since every time a particular name is encountered, it maps to the same replacement.

\subsection{Automated De-Identification of Free-Text Medical Records - Ishna Neamatullah}

The process of de-identification involves [19] scanning the medical notes line-by-line, dividing them into individual words separated by whitespace. First, the algorithm performs a lexical 
match on each word $\mathrm{n}$ the text with dictionaries of PHI look-up tables to locate known and potential PHI, which is then labeled with the associated dictionary type. Second, the algorithm performs pattern matching using regular expressions that look for patters with various context keywords, known as name or location indicators, to find more named entities. Finally, the deidentification process involves replacing each PHI with a tag to indicate to corresponding category.

\subsection{Anonymizing Unstructured Data - Motwani}

We consider the problem of anonymizing datasets in which each individual is associated with a set of items that constitute private information [20] about the individual. One simple way to achieve k-anonymity for a dataset would be to simply remove every item from every user's set, or to add every item from the universe of items to every single set. Naturally this would radically distort the dataset rendering it useless for analyses. So instead, to provide greater utility than such a simplistic scheme, we seek to make the minimal number of changes possible to the dataset in order to achieve the anonymity requirements.

\section{PROPOSED WORK}

Data anonymization consists of treating personal data in such a way that, once the procedure is finished, the data can no longer be used to identify any individual, neither directly nor indirectly, while the value and usefulness of the original data is preserved.

Anonymization Techniques and Standards been designed to successfully anonymize data and avoid identification risks.

\subsection{Text Anonymization Framework}

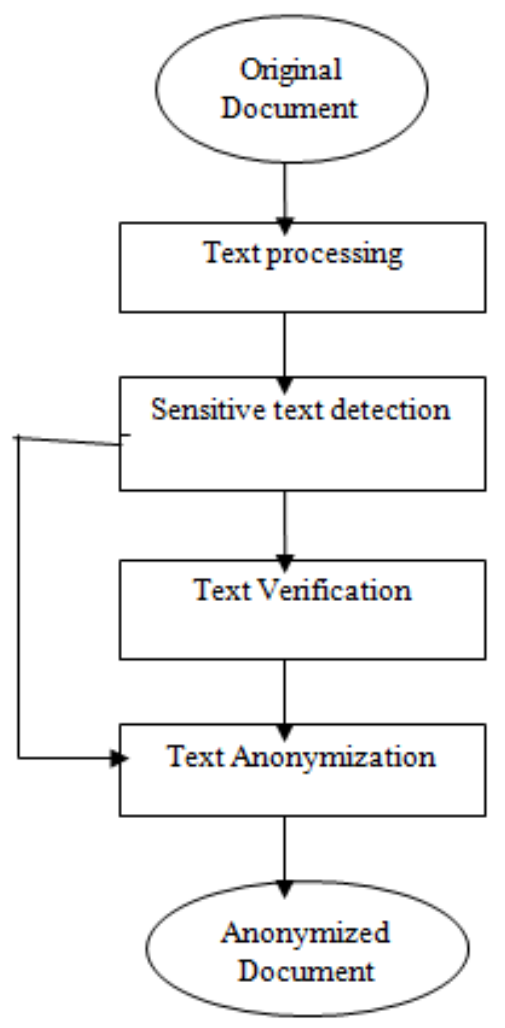

Figure 1 Flowchart of text anonymization process implemented in the proposed work

The text anonymization system shown in Fig 1 is proposed here which is composed of a sequence of four processes: Text-processing, Sensitive text detection, Text verification, and 
Text Anonymization as shown in the figure 1. A text document is received as an input for this text processing module, and returns an anonymized document as an output.

\subsubsection{Text-Processing}

This module prepares for the text in the input document. The text is normalized, split into sentences and then divided in tokens.

\subsubsection{Sensitive Text Detection}

This module uses the function of the NER function to discover the sensitive information in the text. The system made use of NER tool in order to identify the sensitive information since: (i) NEs provide the most feasible data to contain personal information; (ii) a NER tool also provides the class information of the entity; and (iii) earlier works in anonymization were based on NER [21, 22]. This system on receiving an input document will produce a set of expected entities present in the document. Each entity can be identified by its position and its class. Our system aims to identify the three main classes of NEs (person, date, and location).

\subsubsection{Text Validation}

The text validations are performed using the CRR module which helps to determine if two NEs in the same document refer to the similar linguistic object. The NEs reveal the entities of concern within a text document. While performing an anonymization process, we identify the entities those reveal the similar object and replace it by different phrase, and serve the information of the original text document. It is essential to replace all the occurrences of NEs that refer to the similar linguistic object by the same common phrase. In the proposed work, we apply the simple rule-based CRR tool to find the co-reference between NEs which makes it convenient to identify the eligible text for further anonymization, so as to mask the text for the specific information concerning the original entities.

\subsubsection{Anonymization}

This module applies an anonymization method to the entities previously detected. Given a list of recognized entities, it removes or replaces these entities by a specific expression and returns an anonymized version of the text. In order to implement some methods of anonymization, the anonymization module also needs to access external tables of entities and knowledge bases. The methods implemented in this paper are presented in the section below. The objective of this module is to conceal the entities from the text in such a way that those entities cannot be re-identified while the text can still be understood by a human reader.

Different techniques in the anonymization are applied to anonymize the text. Here we have chosen the most frequently used anonymization methods like randomisation, generalization, suppression, substitution and tagging.

- Randomisation: this technique involves data alteration so that the link between the data and the data subject is removed without losing its actual value. The techniques incorporated in this context are: 1) noise addition, adding random small changes in the data; 2) permutation, swapping certain attribute values across individuals, and 3) differential privacy, inserting more or less noise considering a specific query by a particular third party while the original data are preserved.

- Generalization: this technique involves diluting the data subjects' attributes or reducing their granularity. There are two subgroups of generalization techniques: 1) aggregation and k-anonymity, generalizing attribute values in such a way that data subjects are grouped with at least other k individuals; and 2) the l-diversity technique, which extends $\mathrm{k}$-anonymity ensuring that every attribute in an equivalence class has at least 1 values, 
and the t-closeness technique, which refines 1-diversity requiring the values to mirror the original distribution of the data.

- Suppression: a nonaligned placeholder substitutes the item to be anonymized, e.g. "XXXXX", "ZENO". It helps to mask the original sensitive information with a set of unidentifiable expressions.

- Substitution or pseudonymization: the text which is identified to be anonymized is substituted by one of the same category (e.g."Robert" can be a substitute for "James"). While in the process of substitution the new replacing item would be randomly extracted from a dictionary, wisely replaced by a text item that shares the same category.

- Tagging

Tagging or categorization: a tag which indicates its entity type or category is used to replace the item to be anonymized, e.g. "ALEXANDER", "NAME123". This method was implemented concatenating the class given by the NER tool and a unique numeric identifier. All the entities that refer to the same object within the document are replaced by the same label, as provided by the output of the CRR module.

\section{RESULTS AND EVALUATION}

\subsection{Input}

The anonymization system was evaluated using a free text unstructured medical records gathered from with 90 documents. It all together comprised of a total of 23,342 tokens.

\subsection{Evaluation Measures}

We used three metrics to evaluate the performance of the proposed model for the recognition of named entities:

- Precision. Number of named entities correctly identified by the classifier divided by the total number of identified named entities diseases:

$$
\text { Precision }=\frac{\mathrm{TP}}{\mathrm{TP}+\mathrm{FP}}
$$

- Recall. Number of named entities correctly identified by the classifier divided by the number of correct named entities in the original dataset:

$$
\text { Recall }=\frac{\mathrm{TP}}{\mathrm{TP}+\mathrm{FN}}
$$

- F1 Score. Harmonic mean of precision and recall :

$$
\text { F1 Score }=\frac{2 \cdot \text { Precision } * \text { Recall }}{\text { Precision }+ \text { Recall }}
$$

$\mathrm{TP}=$ count of sensitive tokens correctly identified

$\mathrm{FN}=$ count of sensitive tokens missed

$\mathrm{FP}=$ count of non-sensitive tokens falsely identified as sensitive

Table 1 Results

\begin{tabular}{|l|l|l|l|}
\hline $\begin{array}{l}\text { Evaluation } \\
\text { scheme }\end{array}$ & Recall & Precision & F1-Score \\
\hline Token & 71.05 & 78.35 & 72.64 \\
\hline
\end{tabular}

Table 1 show the results for the token anonymization task. We report recall, precision and F1 under all three evaluation schemes. We have developed our own evaluation scripts for the token-based scheme. 


\subsection{Discussion}

The performance of text anonymization using the generalization method only depends on the performance of the NER module and does not take into account the categorization of the NEs into different classes. Generalization does not maintain the information, and the context around the NEs may be insufficient for a clear understanding of the text's content, as it was confusing for a human reader to resolve references between anonymized NEs In order to ensure better anonymization, the NER module must achieve an higher recall. The tagging method enables the reader to resolve references between NEs. Although the replacement tag may allow a reader to identify the class of the anonymized entity, the result does not resemble a natural language text. The performance of this module is dependent on the performance of the NER and CRR modules. The CRR module has been shown to run with an acceptable performance, however some resolution errors occur due to inconsistent classification of entities that mention the same object. Forth at reason, it is important to increase both precision and recall of NER classification module in order to improve the performance of this anonymization method. The random substitution method provides a simple solution for anonymization with a natural language output. However, the relevance of the replacements was low because entities were chosen randomly, so they were often replaced by an entity of a different type (e.g. replacing a street name by a country name), which may cause drifts in the meaning of the text. One possible way of improving the relevance of this method is to use a carefully selected list of replacement entities that are vague enough, in order to avoid semantic drifts. The generalization method provides an output similar to a natural language text. Entities are replaced by a superclass, resulting in a considerably higher relevance than that of the random substitution method. Entities that designate locations achieved a good relevance score, as they are easier to query in the knowledge base. Entities that designate organizations usually raise NE linking issues because an entity may have several entries in the knowledge base with the same name.

\subsection{Output}

Output samples for each anonymization method

\subsubsection{Original}

Raina Angelina, gender female, from Delhi born on 10/05/1983 was diagnosed for Infiltrating Ductal Carcinoma Morphology favours Muccinous Carcinoma - Breast on 26/05/2010. She was under the treatment of Dr George Royce. The shows Estrogen Receptor moderately positive with H-Score 140, Progestrone Receptor negative, HER 2 negative, KI67 Antegen $<10 \%$. She underwent BCS on 10/06/2010 and the received BCS specimen measured $7 \mathrm{X} 6.5 \mathrm{X} 3 \mathrm{~cm}$. on cutting through the identified tumor measuring $3 \mathrm{X} 1.5 \mathrm{X} 6 \mathrm{~m}$ which was grey white, hard, ill circumscribed tumor is at a distance of $1 \mathrm{~cm}$ from superior margin, $1.8 \mathrm{~cm}$ from lateral margin, $1.5 \mathrm{~cm}$ from lateral margin and $1 \mathrm{~cm}$ from anterior margin. FR, FR7 sections studied from superior margin is free from tumor. Clearance is $1 \mathrm{~cm}$. FR2, FR3, FR4, FR5 Section show fibro adipose tissue with breast parenchyma. Section studied is free of tumor. FR6 section studied shows muscle bundles with no tumor.

\subsubsection{Suppression}

XXXXX, gender female, from XXXXX born on XXXXX, was diagnosed for Infiltrating Ductal Carcinoma Morphology favors Muccinous Carcinoma - Breast on XXXXX. She was under the treatment of XXXXX. The reports shows Estrogen Receptor moderately positive with H-Score 140, Progestrone Receptor negative, HER 2 negative, KI67 Antegen <10\%. She underwent BCS on XXXXX and the received BCS specimen measured $7 X 6.5 X 3 \mathrm{~cm}$. on cutting through the identified tumor measuring $3 \times 1.5 \times 6 \mathrm{~m}$ which was grey white, hard, ill 
circumscribed tumor is at a distance of $1 \mathrm{~cm}$ from superior margin; $1.8 \mathrm{~cm}$ fections studied from superior margin is free from tumor. Clearance is $1 \mathrm{~cm}$. FR2, FR3, FR4, FR5 section show fibro adipose tissue with breast parenchyma. Section studied is free of tumor. FR6 section studied shows muscle bundles with no tumor.

\subsubsection{Tagging}

[**PERSON**], gender female, from [**LOCATION] born on [**DATE**], was diagnosed for Infiltrating Ductal Carcinoma Morphology favours Muccinous Carcinoma - Breast on [**DATE**]. She was under the treatment of Dr [**PERSON**]. The reports shows Estrogen Receptor moderately positive with H-Score 140, Progestrone Receptor negative, HER 2 negative, KI67 Antegen $<10 \%$. She underwent BCS on [**DATE**], and the received BCS specimen measured $7 \times 6.5 \times 3 \mathrm{~cm}$. on cutting through the identified tumor measuring $3 \times 1.5 \mathrm{X} 6 \mathrm{~m}$ which was grey white, hard, ill circumscribed tumor is at a distance of $1 \mathrm{~cm}$ from superior margin, $1.8 \mathrm{~cm}$ fections studied from superior margin is free from tumor. Clearance is $1 \mathrm{~cm}$. FR2, FR3, FR4, FR5 ection show fibro adipose tissue with breast parenchyma. Section studied is free of tumor. FR6 section studied shows muscle bundles with no tumor.

\subsubsection{Generalization}

[**Melina Anto**], gender female, from [INDIA] born on [**1981-1990**], was diagnosed for Infiltrating Ductal Carcinoma Morphology favours Muccinous Carcinoma - Breast on [**2011-2020**]. She was under the treatment of Dr [**Naran Varma**]. The reports shows Estrogen Receptor moderately positive with H-Score 140, Progestrone Receptor negative, HER 2 negative, KI67 Antegen $<10 \%$. She underwent BCS on [**2011-2020**], and the received BCS specimen measured $7 \times 6.5 \times 3 \mathrm{~cm}$. on cutting through the identified tumor measuring $3 \times 1.5 \times 6 \mathrm{~m}$ which was grey white, hard, ill circumscribed tumor is at a distance of $1 \mathrm{~cm}$ from superior margin, $1.8 \mathrm{~cm}$ section studied from superior margin is free from tumor. Clearance is $1 \mathrm{~cm}$. FR2, FR3, FR4, FR5 section show fibro adipose tissue with breast parenchyma. Section studied is free of tumor. FR6 section studied shows muscle bundles with no tumor.

\subsubsection{Randomization}

[**Melina Anto**], gender female, from [**Madras**] born on [**01/01/1989**], was diagnosed for Infiltrating Ductal Carcinoma Morphology favours Muccinous Carcinoma Breast on $\left[* * 01 / 11 / 2013^{* *}\right]$. She was under the treatment of Dr [**Naran Varma**]. The reports shows Estrogen Receptor moderately positive with H-Score 140, Progestrone Receptor negative, HER 2 negative, KI67 Antegen $<10 \%$. She underwent BCS on [**15/04/2015**], and the received BCS specimen measured $7 \times 6.5 \times 3 \mathrm{~cm}$. on cutting through the identified tumor measuring $3 \times 1.5 \times 6 \mathrm{~m}$ which was grey white, hard, ill circumscribed tumor is at a distance of $1 \mathrm{~cm}$ from superior margin, $1.8 \mathrm{~cm}$ section studied from superior margin is free from tumor. Clearance is $1 \mathrm{~cm}$. FR2, FR3, FR4, FR5 section show fibro adipose tissue with breast parenchyma. Section studied is free of tumor. FR6 section studied shows muscle bundles with no tumor.

\subsection{Output Sample with Co-Reference Between Entities}

\subsubsection{Original}

Ragav, gender male from Mumbai born on 15/07/1975 was diagnosed for his initial staging of Type 1 Diabetes, who underwent treatment under Dr Desai. Ragav was first diagnosed at the age of 37 years at Kanpur. He was further treated for dengue at the age of 38 and underwent treatment in one of the hospitals at Mumbai. 


\subsubsection{Anonymized}

[**PERSON01**], gender male from [**LOCATON01**] born on [**DATE01**] was diagnosed for his initial staging of Type 1 Diabetes, who underwent treatment under Dr [**PERSON02**]. [**PERSON01**] was first diagnosed at the age of 37 years at [**LOCATON02**]. He was further treated for dengue at the age of 38 and underwent treatment in one of the hospitals at [**LOCATON01**].

Person names were replaced by its super-class entity, change the location and date for a generalized name and date, using the generalization method. The similar objects or entities in the document are replaced with the same responsive entities so as to make the generated anonymized report more convenient for the researcher in understanding the report document much better without any ambiguity.

\section{CONCLUSIONS}

In this paper, we present an implementation of an unstructured data anonymization system of text documents. Different variety of unstructured text documents were used to test this system, using the four different methods of anonymization. The use of the suppression method seems to be efficient as a simple anonymization method, yet it removes relevant semantic information from the text. The tagging method is able to keep some of the information and the co-referential integrity of the mentions to the entities throughout the text. The method of random substitution makes the text have a more natural appearance to a human reader, but most of the times it results in semantic drifts because the entities are chosen randomly from a list. The generalization method presents a more acceptable solution to text anonymization while keeping the appearance natural to a human reader. The performance of this system cannot be compared with the performance of previous systems because the evaluation used different datasets and test conditions. Most of the evaluation of previous anonymization systems aimed at the performance of the NER tool, while this work considered also the performance of the co-reference resolution and anonymization methods.

\section{FUTURE SCOPE}

Future work related to the NER module will aim to improve the performance of the current NER tool and compare the performance of the anonymization system using other NER tools already developed.

\section{REFERENCES}

[1] P'erez-La'inez, R., Iglesias, A., de Pablo-S'anchez, C.: Anonimytext: anonymization of unstructured documents. Universidad Carlos III de Madrid (2009). https:// earchivo.uc3m.es/handle/10016/ 19829

[2] United Kingdom Data Service: Text Anonymization Helper Tool. https:// bitbucket.org/ukda/ukds. tools.textanonhelper/wiki/Home. Accessed 24 Mar 2018

[3] Vico, H., Calegari, D.: Software architecture for document anonymization. Electron. Notes Theor. Comput. Sci. 314(C), 83-100 (2015)

[4] Drechsler, J.: Synthetic Datasets for Statistical Disclosure Control. LNS, vol. 201. Springer, New York (2011). https://doi.org/ 10.1007/978-1-4614-0326-5

[5] Finkel, J.R., Grenager, T., Manning, C.: Incorporating non-local information into information extraction systems by Gibbs sampling. In: Proceedings of the 43rd Annual Meeting of the 
Association for Computational Linguistics (ACL 2005), pp. 363-370. Association for Computational Linguistics (2005)

[6] J. Gardner and L. Xiong, "HIDE: An integrated system for Health Information DEidentification," in Computer-Based Medical Systems. IEEE Computer Society, June 2008, pp. 254-259.

[7] EU General Data Protection Regulation, 2016/679. https://gdpr-info.eu

[8] Kleinberg, B., Mozes, M., van der Toolen, Y., Verschuere, B.: NETANOS - Named Entitybased Text Anonymization for Open Science. Open Science Framework, 31 January 2018. https://osf.io/w9nhb

[9] Domingo-Ferrer, J., S’anchez, D., Soria-Comas, J.: Database Anonymization: Privacy Models, Data Utility, and Micro aggregation-Based Inter-model Connections. Morgan \& Claypool, San Rafael (2016)

[10] Babych, B., Hartley, A: Improving machine translation quality with automatic named entity recognition. In: Proceedings of the 7th International EAMT Workshop on MT and Other Language Technology Tools, Improving MT Through Other Language Technology Tools: Resources and Tools for Building MT (EAMT 2003), pp. 1-8. Association for Computational Linguistics (2003)

[11] Neamatullah, M. Douglass, L. Lehman, A. Reisner, M. Villarroel, W. Long, P. Szolovits, G. Moody, R. Mark, and G. Clifford, "Automated de-identification of free-text medical records," BMC Medical Informatics and Decision Making, vol. 8, no. 1, pp. 1-17, 2008

[12] Zhou, G., Su, J.: Named entity recognition using an HMM-based chunk tagger. In: Proceedings of the 40th Annual Meeting of the Association for Computational Linguistics (ACL 2002), pp. 473-480. Association for Computational Linguistics (2002).

[13] Morwal, S., Jahan, N., Chopra, D.: Named entity recognition using hidden Markov model (HMM). Int. J. Nat. Lang. Comput. 1(4), 15-23 (2012) 17. Nadeau, D., Sekine, S.: A survey of named entity recognition and classification. Linguisticae Investigations 30(1), 3-26 (2007)

[14] Lafferty, J., McCallum, A., Pereira, F.C.N.: Conditional random fields: probabilistic models for segmenting and labeling sequence data. In: Proceedings of the 18th International Conference on Machine Learning 2001 (ICML 2001), pp. 282-289. ACM (2001)

[15] Hundepool, A., et al.: Statistical Disclosure Control. Wiley, New York (2012)

[16] Khalid, M.A., Jijkoun, V., de Rijke, M.: The impact of named entity normalization on information retrieval for question answering. In: Macdonald, C., Ounis, I., Plachouras, V., Ruthven, I., White, R.W. (eds.) ECIR 2008. LNCS, vol. 4956, pp. 705-710. Springer, Heidelberg (2008). https://doi.org/10.1007/ 978-3-540-78646- 783

[17] Ekbal, A., Haque, R., Bandyopadhyay, S.: Bengali part of speech tagging using conditional random field. In: Proceedings of the Seventh International Symposium on Natural Language Processing (SNLP 2007) (2007)

[18] Sweeney, L.: Replacing personally-identifying information in medical records, the Scrub system. In: Proceedings of the AMIA Annual Fall Symposium, p. 333. American Medical Informatics Association (1996) 
[19] Neamatullah, I., et al.: Automated de-identification of free-text medical records. BMC Med. Inform. Decis. Making 8(1), 32 (2008)

[20] Motwani, R. and Shubha U. Nabar. "Anonymizing Unstructured Data.” ArXiv abs/0810.5582 (2008): n. pag.

[21] B. Wellner, M. Huyck, S. Mardis, J. Aberdeen, A. Morgan, L. Peshkin, Y. A., J. Hitzeman, and J. Hirschman, "Rapidly retargetable approaches to de-identification in medical records," Journal of the American Medical Informatics Association: JAMIA, vol. 14, no. 5, pp. 564-573, 2007.

[22] Özlem Uzuner, PhD, Yuan Luo, Peter Szolovits, PhD, Evaluating the State-of-the-Art in Automatic De-identification, Journal of the American Medical Informatics Association, Volume 14, Issue 5, September 2007, Pages 550-563, https://doi.org/10.1197/jamia.M2444 